Anders Bondeson

Thomas Rylander

Pär Ingelström

\title{
Computational Electromagnetics
}

With 74 Illustrations 


\section{Contents}

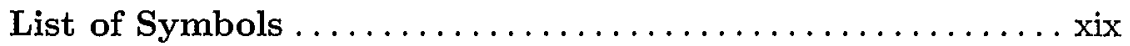

List of Acronyms $\ldots \ldots \ldots \ldots \ldots \ldots \ldots \ldots \ldots \ldots \ldots \ldots \ldots$

1 Introduction $\ldots \ldots \ldots \ldots \ldots \ldots \ldots \ldots \ldots \ldots \ldots \ldots \ldots \ldots \ldots \ldots \ldots$

1.1 Computational Electromagnetics $\ldots \ldots \ldots \ldots \ldots \ldots \ldots \ldots \ldots, 2$

1.2 Maxwell's Equations ........................... 3

1.2.1 Boundary Conditions ..................... 4

1.2.2 Energy Relations ........................ 5

1.2.3 Time Evolution ...................... 6

1.2.4 Dispersion Relation and Wave Velocities .......... 7

1.2.5 Low-Frequency Approximation ................ 8

1.2 .6 Integral Formulation ...................... 9

2 Convergence............................... 11

2.1 Extrapolation to Zero Cell Size.................... 12

2.1.1 A Singular Problem ....................... 15

2.2 Practical Procedures $\ldots \ldots \ldots \ldots \ldots \ldots \ldots \ldots \ldots \ldots \ldots \ldots$

$3 \quad$ Finite Differences ........................... 19

3.1 A 2D Capacitance Problem ...................... 20

3.1.1 Iterative Solution of Laplace's Equation ........... 21

3.1 .2 Computing the Capacitance . ................. 22

3.1.3 MATLAB: Capacitance of Coaxial Cable .......... 23

3.2 Finite Difference Derivatives of Complex Exponentials . . . . . 27

3.2.1 First-Order Derivative ................... 27

3.2.2 Spurious Solutions and Staggered Grids ........... 28

3.2.3 Second-Order Derivative . .................. 30 


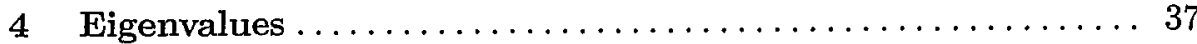

4.1 Maxwell's Equations .......................... 37

4.2 Model Problems............................ 38

4.3 Frequency-Domain Eigenvalue Calculation ............. 39

4.3.1 MATLAB: The 1D Helmholtz Equation ........... 39

4.4 Time-Domain Eigenvalue Calculation . . . . . . . . . . . . . 42

4.4.1 Stability Analysis . ...................... 43

4.4.2 MATLAB: The 1D Wave Equation ............. 45

4.4.3 Extracting the Eigenfrequencies ............... 47

4.4.4 MATLAB: Padé Approximation .............. 50

5 The Finite-Difference Time-Domain Method .......... 57

5.1 The 1D Wave Equation ......................... 58

5.1 .1 Dispersion and Stability .................. 59

5.2 The FDTD Method: Staggered Grids $\ldots \ldots \ldots \ldots \ldots \ldots \ldots \ldots 63$

5.2 .1 One Space Dimension ...................... 63

5.2 .2 Three Space Dimensions $\ldots \ldots \ldots \ldots \ldots \ldots \ldots \ldots 65$

5.2.3 MATLAB: Cubical Cavity ................... 68

5.2.4 Integral Interpretation of the FDTD Method........ 72

5.2.5 Dispersion Analysis in Three Dimensions .......... 75

5.3 Boundary Conditions for Open Regions . . . . . . . . . . . 79

5.3.1 The Perfectly Matched Layer ................. 79

5.3.2 Near-to-Far-Field Transformation . . . . . . . . . . 81

6 The Finite Element Method $\ldots \ldots \ldots \ldots \ldots \ldots \ldots \ldots \ldots, 87$

6.1 General Recipe ............................. 88

6.2 1D Finite Element Analysis . . . . . . . . . . . . . . . . . . 89

6.3 2D Finite Element Analysis ..................... 92

6.3.1 The Assembling Procedure .................. 95

6.3.2 Unstructured Meshes in Practice .............. 99

6.3.3 MATLAB: 2D FEM Using Nodal Basis Functions ..... 100

6.4 Adaptivity .................................... 104

6.5 Vector Equations ........................... 106

6.5.1 Mixed-Order FEM for Systems of First-Order

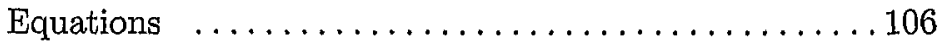

6.5.2 The Curl-Curl Equation and Edge Elements ....... 108

6.5.3 Edge Elements on Cartesian Grids ................ 110

6.5.4 Eigenfrequencies of a Rectangular Cavity ...........113

6.5.5 Edge Elements on Triangles .................. 121

6.5.6 Edge Elements in Practice .................... 123

6.5.7 MATLAB: FEM with Triangular Edge Elements . . . . . 123

6.5.8 Time-Dependent Problems . . ................. 130

6.6 Magnetostatics and Eddy Current Problems ............. 132

6.6.1 2D Formulation ........................... 133

6.6.2 A 2D Application Problem .................. 133 
6.6.3 3D Eddy Current Calculations ................. 136

6.7 Variational Methods ............................ 139

6.7.1 Relation Between Linear Differential Equations and Quadratic Forms ......................... 139

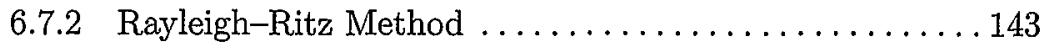

6.7.3 Galerkin's Method........................... 143

6.7.4 A Variational Method for Maxwell's Equations ........ 144

7 The Method of Moments ........................ 153

7.1 Integral Formulation of Electrostatics . . . . . . . . . . 153

7.1.1 Green's Function ........................ 154

7.1.2 General Formulation ..................... 156

7.1.3 FEM Solution.......................... 157

7.2 Capacitance Problem in an Unbounded 2D Region........ 159

7.2 .1 Integration . . . . . . . . . . . . . . . . . . . . 160

7.2.2 MATLAB: MoM for General, 2D Geometries ........ 161

7.2 .3 Charge Distribution ...................... 165

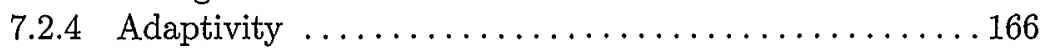

7.2 .5 Numerical Integration....................... 168

7.3 Electromagnetic Scattering . ................... 170

7.3.1 Representation by Potentials and a Lorentz Gauge..... 170

7.3.2 Green's Function for the Vector Potential............. 171

7.3.3 The Electric Field Integral Equation .............. 173

7.3.4 The Magnetic Field Integral Equation............ 176

7.3.5 The Combined Field Integral Equation ............ 177

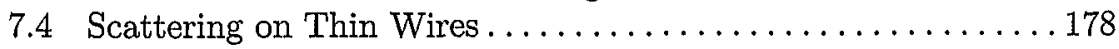

7.4.1 Hallén's Equation ......................... 179

7.4.2 Valid Approximation for the 1D Kernel ............ 180

7.4 .3 Numerical Solution ........................ 181

7.4.4 MATLAB: Hallén's Equation . . . . . . . . . . . . . 181

7.4.5 Numerical Results ........................ 184

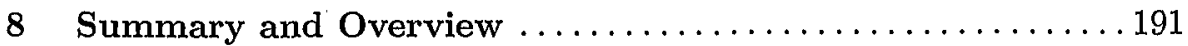

8.1 Differential Equation Solvers ........................ 192

8.1.1 Finite-Difference Time-Domain.................. 193

8.1 .2 Finite-Volume Time-Domain . . . . . . . . . . . . . . . 193

8.1.3 Finite Element Method ....................... 194

8.1.4 Transmission Line Method...................... 194

8.1.5 Finite Integration Technique . . . . . . . . . . . . . 195

8.2 Integral Equation Solvers ....................... 195

8.2.1 Frequency-Domain Integral Equations............. 196

8.2.2 Time-Domain Integral Equations . . . . . . . . . . . . 197

8.3 Hybrid Methods............................ 198 
xviii Contents

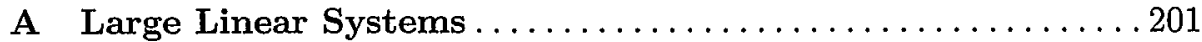

A.1 Sparse Matrices . . . . . . . . . . . . . . . . . . . 201

A.2 Solvers for Large Sparse Systems of Equations . . . . . . . . . . 204

A.2.1 Direct Solvers . . . . . . . . . . . . . . . . . . . 204

A.2.2 Iterative Solvers . . . . . . . . . . . . . . . . . . . . . 204

A.2.3 Multigrid Methods . . . . . . . . . . . . . . 205

A.3 Capacitance Calculation on Larger Grids . . . . . . . . . . . . 206

B Krylov Methods ..............................209

B.1 Projection Methods......................... 209

B.2 Krylov Methods .......................... 210

B.3 Nonsymmetric A . . . . . . . . . . . . . . . . 211

B.4 Preconditioning . . . . . . . . . . . . . . . . . 212

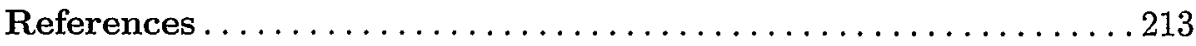

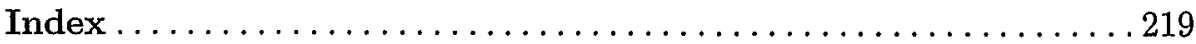

